\title{
Subband-Adaptive Turbo-Coded OFDM-Based Interactive Video Telephony
}

\author{
Peter J. Cherriman, Thomas Keller, and Lajos Hanzo
}

\begin{abstract}
A range of adaptive orthogonal frequency division multiplex (AOFDM) video systems are proposed for interactive communications over wireless channels. The proposed constant target bit-rate subband adaptive OFDM modems can provide a lower bit error rate than a corresponding conventional OFDM modem. The slightly more complex switched or time-variant target bit rate AOFDM modems can provide a balanced video quality performance, across a wider range of channel signal-to-noise ratios, maintaining the best video performance. Upon invoking the technique advocated-irrespective of the channel conditions experienced-the transceiver achieves always the best possible video quality by automatically adjusting the achievable bit rate and the associated video quality in order to match the channel quality experienced. This is achieved on a near-instantaneous basis under given propagation conditions in order to cater for the effects of path-loss, fast-fading, slow-fading, dispersion, etc. Furthermore, when the mobile is roaming in a hostile outdoor propagation environment, typically low-order, low-rate modem modes are invoked, while in benign indoor environments predominantly the high-rate, high source-signal representation quality modes are employed.
\end{abstract}

Index Terms-Adaptive AOFDM, adaptive transceiver, AOFDM, H.263 video codec, OFDM, wireless video telephony.

\section{INTRODUCTION}

A. State-of-the-Art in Near-Instantaneously Adaptive Transceivers

B URST-BY-BURST (BbB) adaptive quadrature amplitude modulation [1], [3], [4] (AQAM) was contrived by Steele and Webb [1], [5], [6], in order for the transceiver to counteract the time-variant channel quality fluctuations of narrow-band fading channels. Further related research was conducted, for example, at the University of Osaka by Sampei and his colleagues, combining adaptive modulation with variable coding rate concatenated channel coded schemes [7]. At the University of Stanford, Goldsmith and her team studied the effects of variable-rate, variable-power arrangements [8]. A range of related research problems were also addressed at the University of Southampton, investigating a variety of practical aspects of AQAM [1], [3], [4], [9], [10]. The channel's near-instantaneously fluctuating quality is estimated on a burst-by-burst basis and the highest throughput modulation mode is selected

Manuscript received August 4, 1999; revised July 16, 2002. This work was supported by the Mobile VCE, EPSRC, U.K., in the framework of the Contract GR/K 74043 and by the European Commission. This paper was recommended by Associate Editor X. Lin.

The authors are with the Department of Electronic and Computer Science, University of Southampton, Southampton SO17 1BJ, U.K. (e-mail lh@ecs.soton.ac.uk).

Digital Object Identifier 10.1109/TCSVT.2002.804887 that is capable of maintaining the required target bit error rate (BER) performance. This is a novel design paradigm, allowing us to maximize the system's bits per symbol (BPS) throughput, hence maximizing the achievable video quality. Using this reconfiguration regime, the distribution of channel errors becomes typically less bursty than in conjunction with nonadaptive modems, which potentially increases the attainable channel coding gain [1], [3], [4]. Furthermore, the soft-decision channel codec output metrics can be also invoked for estimating the instantaneous channel quality [1], [3], [4], irrespective of the type of channel impairments.

To elaborate a little further on the advances in AQAM, a range of coded AQAM schemes were analyzed by Matsuoka et al. [7], Lau et al. [11], and Goldsmith et al. [12]. For data transmission systems which do not necessarily require a low transmission delay, variable-throughput adaptive schemes can be devised, which operate efficiently in conjunction with powerful error correction codecs, such as long block-length turbo codes [36], [37]. However, the acceptable turbo interleaving delay is rather low in the context of low-delay interactive speech. Video communications systems [4] typically require a higher bit rate than speech systems [22], and hence they can afford a higher interleaving depth expressed in terms of the number of bits, without imposing a long delay.

The above AQAM principles-which were typically investigated in the context of narrowband modems in the early phase of research-were then further advanced in conjunction with wideband modems, employing powerful block turbo coded wideband decision feedback equalizer (DFE)-assisted AQAM transceivers [3]. As a design alternative, a neural-network assisted so-called radial basis function (RBF) DFE-based AQAM modem design was proposed in [3], where the RBF DFE provided the channel quality estimates for the AQAM modem's mode-switching regime. This RBF-aided modem was capable of removing the residual BER of conventional DFEs, even when linearly nonseparable received phasor constellations were encountered due to the dispersive fading channel.

The above burst-by-burst adaptive principles can also be extended to adaptive orthogonal frequency division multiplexing (AOFDM) schemes [1], [2] and to adaptive joint-detection based code division multiple access (JD-ACDMA) arrangements [3]. The focus of this contribution is AOFDM-assisted interactive video telephony, hence we elaborate a little further on the various advances in the field of AOFDM. Specifically, the associated AQAM principles were invoked in the context of parallel AOFDM modems also by Czylwik et al. [27], Fischer [28] and Chow et al. [29]. Adaptive subcarrier selection has 
been advocated furthermore by Rohling et al. [34] in order to achieve BER performance improvements. Further significant advances are due to Chow et al. [29] from the U.S. Their research efforts rendered OFDM the dominant solution for asymmetric digital subscriber loop (ADSL) applications, potentially supporting bit rates up to $54 \mathrm{Mbits} / \mathrm{s}$, when communicating over benign, slowly varying dispersive Gaussian fixed links, rather than over hostile wireless links. In Europe, OFDM has been favored for both digital audio broadcasting (DAB) and digital video broadcasting [14], [15] (DVB) as well as for high-rate Wireless Asynchronous Transfer Mode (WATM) systems due to its ability to combat the effects of highly dispersive channels [16]. The idea of "water-filling"-as allocating different modem modes to different subcarriers was referred to-was proposed for OFDM by Kalet [33] and was further advanced later by Chow et al. [29]. This approach was then developed for transmission over time-variant duplex wireless links in [1]. In the field of applications, a high-quality OFDM-based speech system was proposed in [22]. Finally, while OFDM is sensitive to the co-channel interference inflicted in wireless communications, this deficiency can be mitigated with the aid of adaptive beam-forming [19]-[21] in multiuser scenarios.

\section{B. Motivation}

The main contribution of this paper is demonstrating with the aid of a turbo-coded system design study that upon invoking the technique advocated-irrespective of the channel conditions experienced - the transceiver achieves always the best possible video quality. This is attained by automatically adjusting the achievable bit rate and the associated video quality in order to match the channel quality experienced. We control the allocation of video bits on a near-instantaneous basis under given propagation conditions in order to counteract the effects of path-loss, fast-fading, slow-fading, dispersion, etc. Furthermore, when encountering a hostile outdoor propagation environment, typically low-order, low-rate modem modes are invoked, while in benign indoor environments, predominantly the high-rate, high source-signal representation quality modes are employed. This is achieved by adaptively controlling the near-instantaneous video bit rate. Similar principles can be also invoked by adaptively mapping speech/audio bits to the OFDM subcarriers, as it was investigated in [22] in the context of the high-quality G.722.1 wideband speech codec, but due to lack of space, these speech/audio transmission issues are beyond the scope of this paper.

The remainder of this contribution is structured as follows. Section II outlines the architecture of the proposed video transceiver, while Section III quantifies the performance benefits of AOFDM transceivers in comparison to conventional fixed transceivers. Section IV endeavors to highlight the effects of more "aggressive" loading of the subcarriers in both BER and video quality terms, while Section $\mathrm{V}$ proposed time-variant, rather than constant rate AOFDM as a means of more accurately matching the transceiver to the time-variant channel quality fluctuations, before concluding in Section VI.

\section{BURST-By-BURST AdAPTIVE VIDEO TRANSCEIVER}

\section{A. AOFDM Modem Mode Adaptation and Signaling}

The proposed duplex AOFDM scheme operates on the following basis.

- Channel quality estimation is invoked upon receiving an AOFDM symbol, in order select the modem mode allocation of the next AOFDM symbol.

- The decision concerning the modem modes for the next AOFDM symbol is based on the prediction of the expected channel conditions. Then the transmitter has to select the appropriate modem modes for the groups or subbands of OFDM subcarriers, where the subcarriers were grouped into subbands of identical modem modes, in order reduce the required number of signaling bits.

- Explicit signaling or blind detection of the modem modes is used to inform the receiver as to what type of demodulation to invoke.

More explicitly, if the channel is reciprocal, then the channel quality estimate for the uplink can be extracted from the downlink and vice versa. We refer to this regime as open-loop adaptation. In this case, the transmitter has to convey the modem modes to the receiver, or the receiver can attempt blind detection of the transmission parameters employed. By contrast, if the channel cannot be considered reciprocal, then the channel quality estimation has to be performed at the receiver and the receiver has to instruct the transmitter as to what modem modes have to be used at the transmitter, in order to satisfy the target integrity requirements of the receiver. We refer to this mode as closed-loop adaptation. Blind modem mode recognition was invoked, for example, in [1] and [3] — a technique, which results in bit-rate savings due to dedicating no bits to explicit modem mode signaling. Naturally, this can be achieved only at the cost of increased complexity. Let us address the issues of channel quality estimation on a subband-by-subband basis in the next subsection.

\section{B. AOFDM Subband BER Estimation}

When OFDM is used for communicating over highly frequency-selective channels, each subcarrier can be allocated a different transmit power and a different modulation mode. This OFDM symbol-by-symbol based "resource" allocation can be optimized with the aid of an algorithm which, if the channel is time variant, has to be repeated on an OFDM symbol-by-symbol basis. Some of the existing algorithms [23], [33] are mainly of theoretical interest due to their high complexity. Among the practical algorithms [24], [25], [27]-[29], the Hughes-Hartog algorithm (HHA) [26], [25] is perhaps best known, but its complexity is somewhat high, especially for real-time OFDM symbol-by-symbol based applications at high bit rates. Hence, the HHA has stimulated extensive research for computationally more efficient algorithms [24], [25], [27]-[29][29]. The most efficient appears to be that of Lai et al. [25], which is a fast version of the HHA and that of Piazzo [24].

In our system, the following modem mode allocation principle was used. A reliable channel quality metric can be devised by calculating the expected overall bit error probability (BEP) of 
$\bar{p}_{e}(n)$ for all available modulation modes $M_{n}$ in each subband. More explicitly, given the channel signal-to-noise ratio (SNR) of $\gamma_{j}$ averaged across the $N_{s}$ number of subcarriers in the $j$ th subband, the expected overall BER for all the available modulation modes $M_{n}$ in each subband can be estimated, which is expressed as $\bar{p}_{e}(n)=1 / N_{s} \sum_{j} p_{e}\left(\gamma_{j}, M_{n}\right)$. For each subband, the modulation mode $M_{n}$ having the highest throughput, whose estimated BER is lower than the target BER is then activated. These issues will be further detailed in Section V. While the adaptation accuracy is limited due to the subband width, the channel quality estimation is quite reliable, even in interference-impaired environments. The results of [1] demonstrate that there is only marginal throughput loss due to using a subband width of 16-32 subcarriers, rather than allowing the modem modes to freely change on a subcarrier-by-subcarrier basis.

Apart from the choice of the modem mode allocation principle, another important issue is the signaling of the subband modem modes. As a design alternative, Otsuki et al. [30] proposed using a four-chip Walsh code for signaling the modem modes, while Torrance and Hanzo [31] advocated the employment of a specific uneven error protection based PSK modulation constellation having five phasors for signaling a set of five AQAM modes, which were nonuniformly distributed on the 5-PSK constellation ring. The performance of various blind mode signaling schemes was documented in [1], [3] under the assumption that, in case of a modem mode signaling error, the BER was $50 \%$. The performance degradations imposed by outdated channel quality estimates arriving after a feedback delay of one transmission burst were also documented for various normalized Doppler frequencies in [1], [3]. These were counteracted by increasing the AQAM mode-switching thresholds for the sake of more cautiously activating the high-BPS AQAM modes. Increasing the switching thresholds slightly reduced the achievable BPS throughput. These effects can be further mitigated using powerful channel-quality prediction techniques [32], [3].

Against this background in our forthcoming discussions the design trade-offs of turbo-coded AOFDM wideband video transceivers are presented. We will demonstrate that AOFDM provides a convenient framework for adjusting the required target integrity and throughput both with and without turbo channel coding and lends itself to attractive video system construction, provided that a near-instantaneously programmable rate video codec — such as the H.263 scheme highlighted in the next section-can be invoked.

\section{Video Compression and Transmission Aspects}

In this study, we investigate the transmission of $704 \times 576$ pixel four-times common intermediate format (4CIF) high-resolution video sequences at 30 frames/s using subband-adaptive turbo-coded AOFDM transceivers. The transceiver can modulate 1,2 , or 4 bits onto each AOFDM subcarrier, or simply disable transmissions for subcarriers which exhibit a high attenuation or phase distortion due to channel effects.

The H.263 video codec [4] exhibits an impressive compression ratio, although this is achieved at the cost of a high vulnerability to transmission errors, since a run-length coded bitstream is rendered undecodable by a single bit error. In order to miti- gate this problem, when the channel codec protecting the video stream is overwhelmed by the transmission errors, we refrain from decoding the corrupted video packet in order to prevent error propagation through the reconstructed video frame buffer [35]. We found that it was more beneficial, in video quality terms, if these corrupted video packets were dropped and the reconstructed frame buffer was not updated until the next video packet replenishing the specific video frame area was received. The associated video performance degradation was found perceptually unobjectionable for video packet loss ratios or transmission frame error ratios (FERs) ${ }^{1}$ below about $5 \%$. This was demonstrated in [35, Fig. 7] and in [4]. ${ }^{2}$ These packet dropping events were signaled to the remote video decoder by superimposing a strongly protected 1-bit packet acknowledgment flag on the reverse-direction packet, as outlined in [35]. Turbo error-correction codes [36], [37] were used. The associated parameters will be discussed in more depth during our further discourse.

\section{COMPARISON OF SUBBAND-ADAPTIVE OFDM AND FIXED-MODE OFDM TRANSCEIVERS}

In order to show the benefits of the proposed subband-adaptive OFDM transceiver, we compare its performance to that of a fixed modulation mode transceiver under identical propagation conditions, while having the same transmission bit rate. The subband-adaptive modem is capable of achieving a low BER, since it can disable transmissions over low quality subcarriers and compensate for the lost throughput by invoking a higher modulation mode, than that of the fixed-mode transceiver over the high-quality subcarriers.

Table I shows the system parameters for the fixed BPSK and QPSK transceivers, as well as for the corresponding AOFDM transceivers. ${ }^{3}$ The system employs constraint length three, half-rate turbo coding, using octal generator polynomials of 5 and 7 [37] as well as random turbo interleavers and four turbo iterations. The turbo interleaver interleaved the video bits over one OFDM symbol, regardless of its useful "payload" expressed in terms of the number of bits per OFDM symbol, which conveniently supported symbol-by-symbol detection and turbo decoding of each OFDM symbol. Therefore, the unprotected bit rate is approximately half the channel coded

\footnotetext{
${ }^{1}$ The terms transmission FER and video packet loss ratio (PLR) are used synonymously in the paper, since the video packets are directly mapped to transmission frames, each constituted by an OFDM symbol.

${ }^{2}$ Under the experimental conditions used, the associated peak SNR (PSNR) degradation at this FER was found to be less than $1 \mathrm{~dB}$. We note, however, that the tolerable FER is a function of the size of the dropped packet as well as that of the resolution of the video frames transmitted. The larger the video frame, the higher the resolution and the more visible the obsolete video frame segments become. The corresponding subjective video degradation effects can be viewed with the aid of an MPEG player at http://www-mobile.ecs.soton.ac.uk/peter/robust-h263/robust.html\#DROPPING.

${ }^{3}$ We note that in single-carrier systems the conventional normalized Doppler frequency is used for quantifying the rate of fading-induced signal envelope fluctuations, which is normalized to the duration of the transmitted symbols. By contrast, in OFDM systems it is more relevant to use the Doppler frequency normalized to the duration of an OFDM symbol, because this characterizes the rate of channel quality fluctuations experienced by the OFDM modem [1]. Furthermore, error-free mode signaling was assumed, while the performance of various blind mode signaling schemes was documented in [1] under the assumption that in case of a modem mode signaling error the BER was $50 \%$.
} 
TABLE I

SYSTEM PARAMETERS FOR THE FIXED QPSK AND BPSK TRANSCEIVERS, AS WELL AS FOR THE CORRESPONDING AOFDM TRANSCEIVERS FOR WLANS

\begin{tabular}{l|c|c}
\hline & BPSK mode & QPSK mode \\
\hline Packet rate & \multicolumn{2}{|c}{4687.5 Packets/s } \\
\hline FFT length & \multicolumn{2}{|c}{512} \\
\hline OFDM symbols/packet & \multicolumn{2}{|c}{3} \\
\hline OFDM symbol duration & \multicolumn{2}{|c}{$2.6667 \mu \mathrm{s}$} \\
\hline OFDM time frame & \multicolumn{2}{|c}{80 Timeslots $=213 \mu \mathrm{s}$} \\
\hline Normalised Doppler frequency, $f_{d}^{\prime}$ & \multicolumn{2}{|c}{$1.235 \times 10^{-4}$} \\
\hline OFDM symbol normalised Doppler frequency, $F_{D}$ & \multicolumn{2}{|c}{$7.41 \times 10^{-2}$} \\
\hline FEC coded bits/packet & 1536 & 3072 \\
\hline FEC-coded video bitrate & $7.2 \mathrm{Mbps}$ & $14.4 \mathrm{Mbps}$ \\
\hline Unprotected Bits/Packet & 766 & 1534 \\
\hline Unprotected bitrate & $3.6 \mathrm{Mbps}$ & $7.2 \mathrm{Mbps}$ \\
\hline Error detection CRC (bits) & 16 & 16 \\
\hline Feedback error flag bits & 9 & 9 \\
\hline Packet header bits/packet & 11 & 12 \\
\hline Effective video bits/packet & 730 & 1497 \\
\hline Effective video bitrate & $3.4 \mathrm{Mbps}$ & $7.0 \mathrm{Mbps}$ \\
\hline
\end{tabular}

bit rate. The protected to unprotected video bit rate ratio is not exactly half, since two tailing bits are required to reset the convolutional encoders' memory to their default state in each transmission burst. In both modes a 16-bit Cyclic Redundancy Checking (CRC) is used for error detection and 9 bits are used to encode the reverse link feedback acknowledgment information by simple repetition coding. The feedback flag decoding ensues using majority logic decisions. The packetization requires a small amount of header information added to each transmitted packet, which is 11 and 12 bits per packet for BPSK and QPSK, respectively. The effective or useful video bit rates for the BPSK and QPSK modes are then 3.4 and 7.0 Mbits/s.

The fixed-mode BPSK and QPSK transceivers are limited to one and two bits per symbol, respectively. By contrast, the proposed AOFDM transceivers operate at the same bit rate, as their corresponding fixed modem mode counterparts, although they can vary their modulation mode on a subcarrier by subcarrier basis between $0,1,2$, and 4 bits per symbol. Zero bits per symbol implies that transmissions are disabled for the subcarrier concerned.

The micro-adaptive nature of the subband-adaptive modem is characterized by Fig. 1, portraying in Fig. 1(a) a contour plot of the channel SNR for each subcarrier versus time. This was recorded for the channel impulse response (CIR) of Fig. 2. In Fig. 1(b) and (c), the modulation mode chosen for each 32-subcarrier subband is shown versus time for the 3.4-and 7.0-Mbits/s target-rate subband-adaptive modems, respectively. As it was augmented in more depth in [1], this CIR was generated with the aid of ray-tracing in a laboratory environment, when experiencing two reflected paths corresponding to a maximum path delay of about $15 \mathrm{~m}$. The path delay expressed in terms of nanoseconds is related to the path length difference expressed in terms of the distance are related to each other by the speed of radio wave propagation, which is the same as the speed of light. A detailed discussion of Fig. 1(d) is provided in Section $\mathrm{V}$ in the context of the time-variant bit-rate mode of the system.
It can be seen that when the channel is of high quality-like, for example, at about frame 1080-the subband-adaptive modem used the same modulation mode, as the equivalent fixed-rate modem in all subcarriers. When the channel is hostile-like around frame 1060 - the subband-adaptive modem used a lower order modulation mode in some subbands than the equivalent fixed-mode scheme, or in extreme cases disabled transmission for that subband. In order to compensate for the loss of throughput in this subband, a higher order modulation mode was used in the higher quality subbands.

One video packet is transmitted per OFDM symbol, therefore the video packet loss ratio is the same, as the OFDM symbol error ratio. The video packet loss ratio is plotted versus the channel SNR in Fig. 3. It is shown in the graph that the subband-adaptive transceivers-or synonymously termed as microscopic-adaptive ( $\mu \mathrm{AOFDM})$, in contrast to OFDM symbol-by-symbol adaptive transceivers-have a lower PLR at the same SNR compared to the fixed modulation mode transceiver. Note in Fig. 3 that the subband-adaptive transceivers can operate at lower channel SNRs than the fixed-modem mode transceivers, while maintaining the same required video packet loss ratio. Again, the figure labels the subband-adaptive OFDM transceivers as $\mu$ AOFDM, implying that the adaption is not noticeable from the upper layers of the system. A macro-adaption could be applied in addition to the microscopic adaption by switching between different target bit rates, as the longer term channel quality improves and degrades. This issue is the subject of Section V.

Having shown that the subband-adaptive OFDM transceiver achieved a reduced video packet loss in comparison to fixed modulation mode transceivers under identical channel conditions, we now compare the effective throughput bit rate of the fixed and adaptive OFDM transceivers in Fig. 4. The figure shows that when the channel quality is high, the throughput bit rate of the fixed and adaptive transceivers are identical. However, as the channel degrades, the loss of packets results in a lower effective throughput bit rate for both the fixed-mode and the adaptive transceiver. However, the lower packet loss 


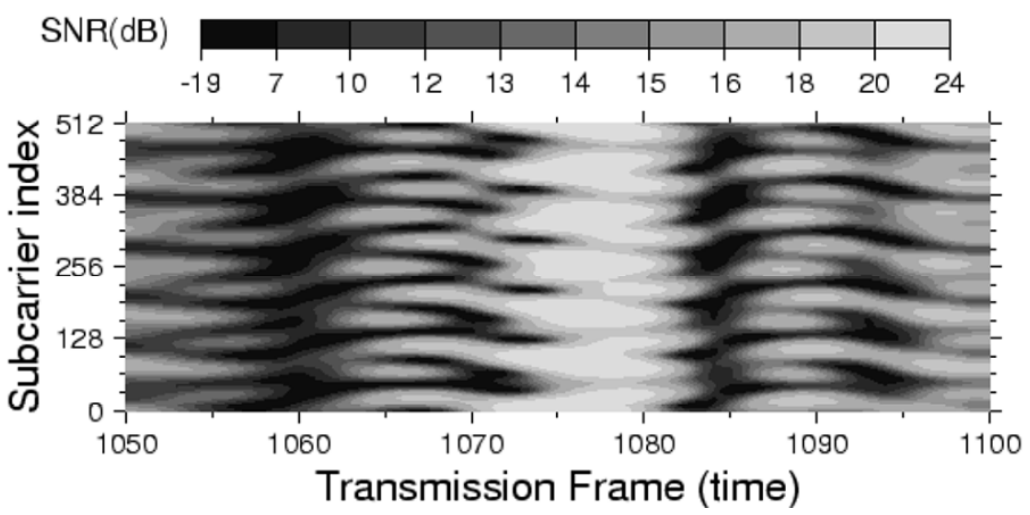

(a)

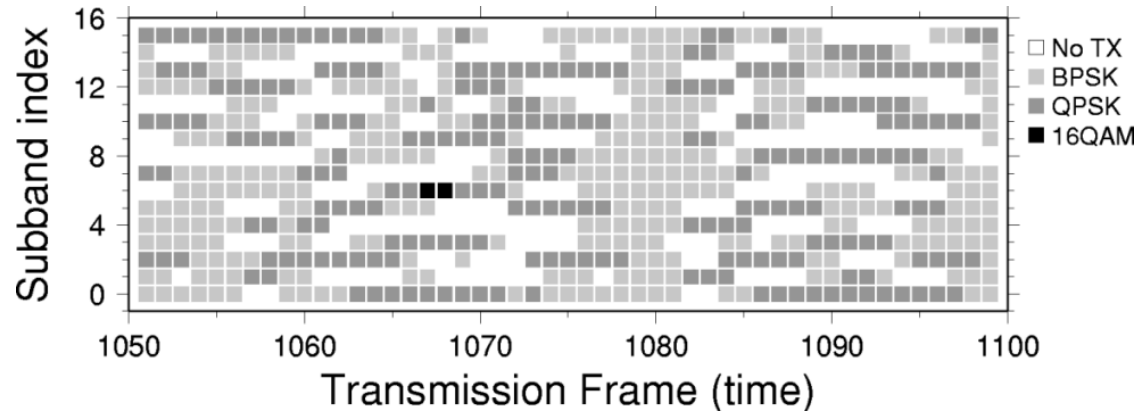

(b)

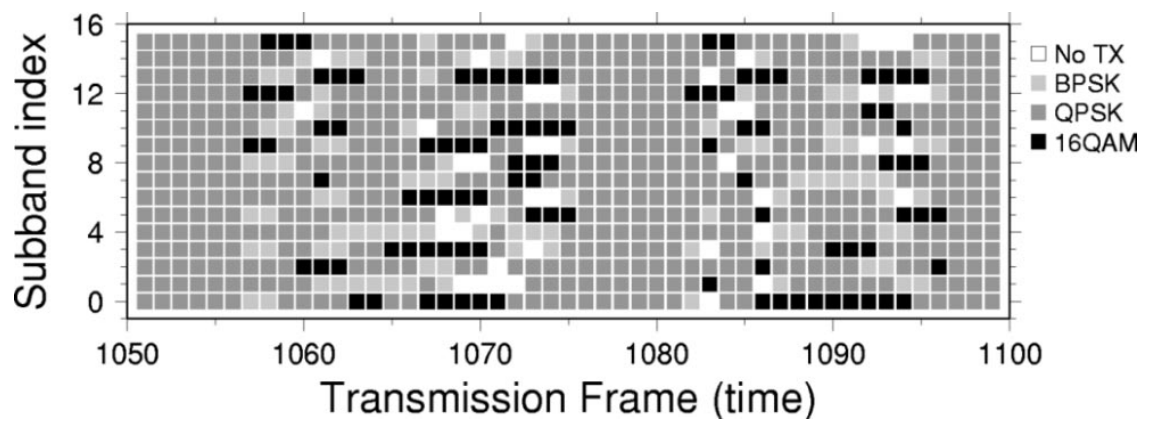

(c)

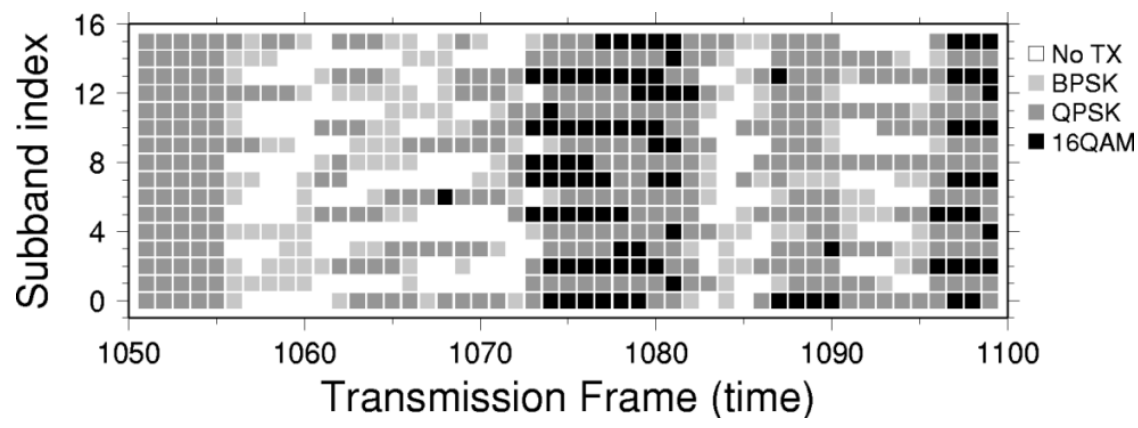

(d)

Fig. 1. Bit allocation in the subband-adaptive OFDM modem. The average channel SNR was $16 \mathrm{~dB}$. (a) Contour plot of the channel SNR for all 512 subcarriers versus time. (b) and (c) show the micro-adaptive constant-bit-rate modulation modes chosen for all 1632 -subcarrier subbands for the same period of time. (b) Performance of the 3.4-Mbits/s subband-adaptive modem, which operates at the same bit rate as a fixed BPSK modem. (c) 7.0-Mbits/s subband-adaptive modem, which operated at the same bit rate as a fixed QPSK modem. (d) Modulation mode chosen for all 16 subbands for the same period of time in the time-variant bit-rate mode of the system, which is the subject of Section V.

ratio of the subband-adaptive transceiver results in a higher throughput bit rate than that of the fixed modulation mode based transceiver.

The throughput bit-rate performance results translate to the decoded video quality performance results evaluated in terms of the PSNR in Fig. 5. Again, for high channel SNRs, the performance of the fixed and adaptive OFDM transceivers is identical. However, as the channel quality degrades, the video quality of the subband-adaptive transceiver degrades less dramatically than that of the corresponding fixed modulation 
Time Delay [ns]

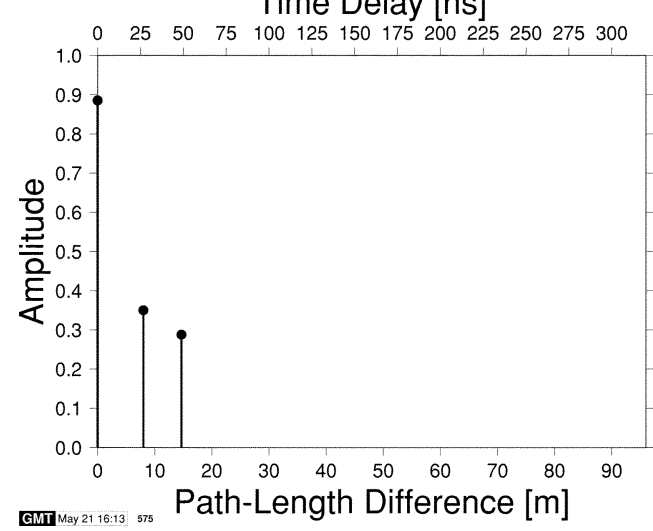

Fig. 2. Indoor three-path WATM channel impulse response.

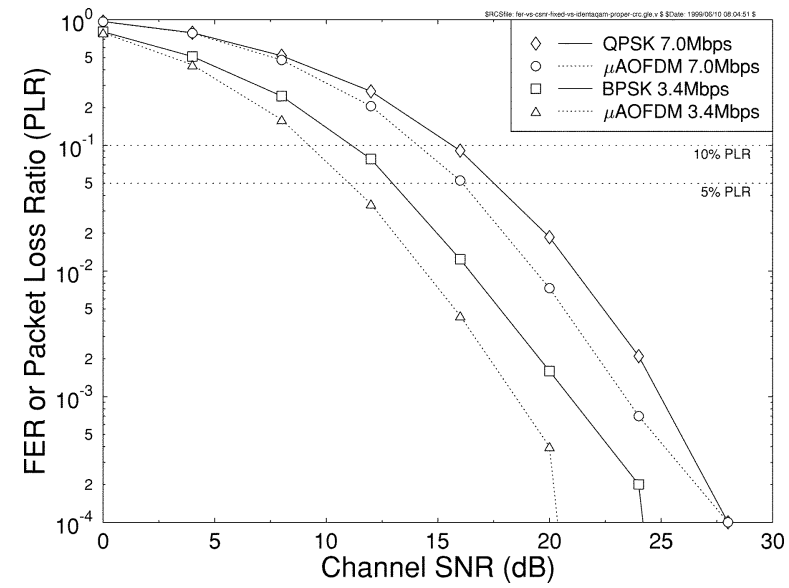

Fig. 3. FER or PLR versus channel SNR for the BPSK and QPSK fixed modulation mode OFDM transceivers and for the corresponding subband-adaptive $\mu \mathrm{AOFDM}$ transceiver, operating at identical effective video bit rates, namely at 3.4 and $7.0 \mathrm{Mbits} / \mathrm{s}$, over the channqel model of Fig. 2 at a normalized Doppler frequency of $F_{D}=7.41 \times 10^{-2}$.

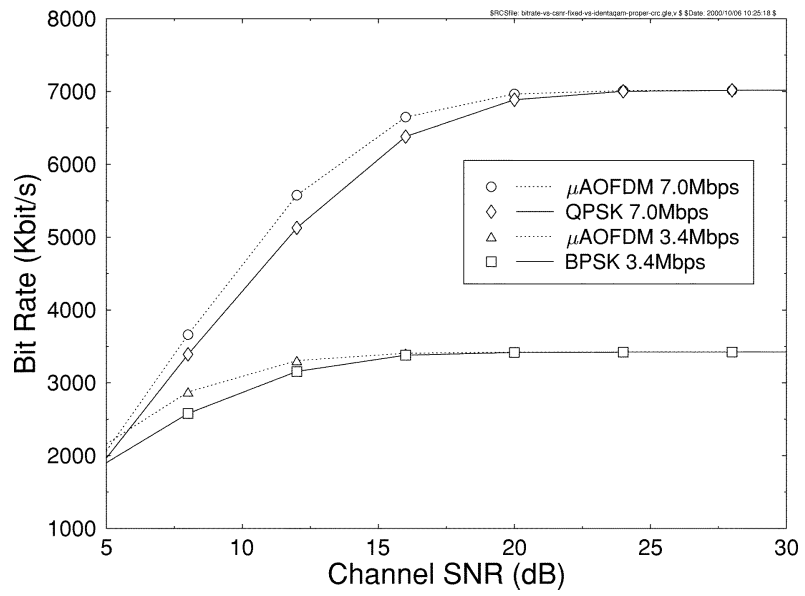

Fig. 4. Effective throughput bit rate versus channel SNR for the BPSK and QPSK fixed modulation mode OFDM transceivers and that of the corresponding subband-adaptive or $\mu \mathrm{AOFDM}$ transceiver operating at identical effective video bit rates of 3.4 and $7.0 \mathrm{Mbits} / \mathrm{s}$, over the channel of Fig. 2 at a normalized Doppler frequency of $F_{D}=7.41 \times 10^{-2}$.

mode transceiver. More explicitly, at a channel SNR of about $20 \mathrm{~dB}$, the adaptive system requires approximately $2-\mathrm{dB}$ lower channel SNR, than the fixed-mode scheme for ensuring the same perceptually unimpaired video quality.

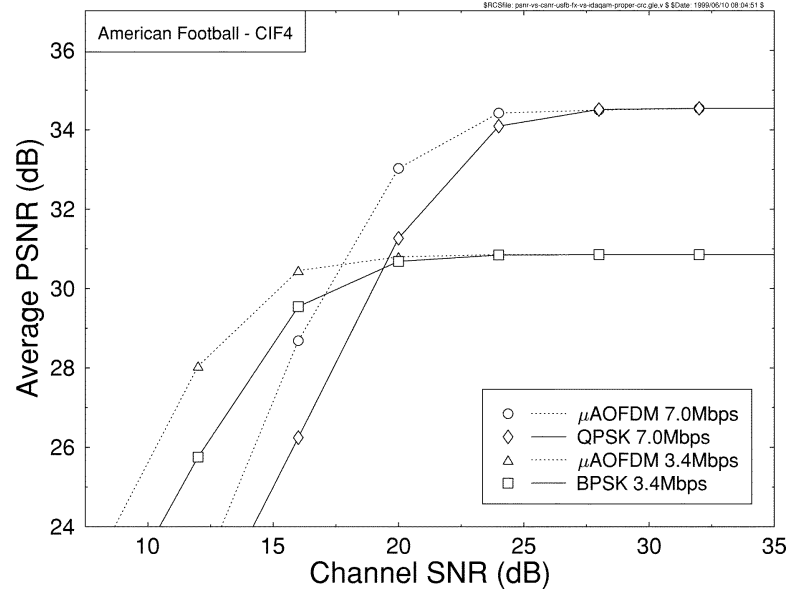

Fig. 5. Average PSNR video quality versus channel SNR for the BPSK and QPSK fixed modulation mode OFDM transceivers and for the corresponding $\mu$ AOFDM transceiver operating at identical channel SNRs over the channel model of Fig. 2 at a normalized Doppler frequency of $F_{D}=7.41 \times 10^{-2}$.

\section{Subband-ADAPTiVE OFDM TRANSCEIVERS HAVING DIFFERENT TARGET BIT RATES}

As mentioned before, the subbannd-adaptive modems employ different modulation modes for different subcarriers, in order to meet the target bit rate requirement at the lowest possible channel SNR. This is achieved by using a more robust modulation mode or eventually by disabling transmissions over subcarriers having a low channel quality. By contrast, the adaptive system can invoke less robust but higher throughput modulation modes over subcarriers exhibiting a high channel quality. In the examples previously considered, we chose the AOFDM target bit rate to be identical to that of a fixed modulation mode based transceiver. In this section, we comparatively study the performance of various $\mu$ AOFDM systems having different target bit rates.

The previously described $\mu$ AOFDM transceiver of Table I exhibited a FEC-coded bit rate of $7.2 \mathrm{Mbits} / \mathrm{s}$, which provided an effective video bit rate of $3.4 \mathrm{Mbits} / \mathrm{s}$. If the video target bit rate is lower than $3.4 \mathrm{Mbits} / \mathrm{s}$, then the system can disable transmission in more of the subcarriers, where the channel quality is low. Such a transceiver would have a lower BER than the previous BPSK-equivalent $\mu \mathrm{AOFDM}$ transceiver, and therefore, could be used at lower average channel SNRs, while maintaining the same BER target. By contrast, as the target bit rate is increased, the system has to employ higher-order modulation modes in more subcarriers, at the cost of an increased BER. Therefore, high target bit rate $\mu$ AOFDM transceivers can only perform within the required BER constraints at high channel SNRs, while low target bit rate $\mu$ AOFDM systems can operate at low channel SNRs without inflicting excessive BERs. Therefore, a system which can adjust its target bit rate as the channel SNR changes would operate over a wide range of channel SNRs, providing the maximum possible average throughput bit rate, while maintaining the required BER.

Hence, below we provide a performance comparison of various $\mu$ AOFDM transceivers having four different target bit rates, of which two are equivalent to that of the BPSK and QPSK fixed 
TABLE II

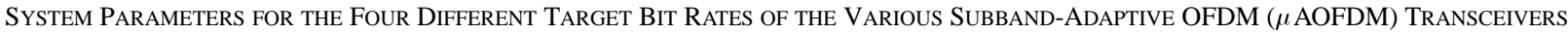

\begin{tabular}{l|c|c|c|c}
\hline Packet rate & \multicolumn{4}{|c}{4687.5 Packets/s } \\
\hline FFT length & \multicolumn{4}{|c}{312} \\
\hline OFDM Symbols/Packet & \multicolumn{4}{|c}{$2.6667 \mu \mathrm{s}$} \\
\hline OFDM Symbol Duration & \multicolumn{4}{|c}{$1.235 \times 10^{-4}$} \\
\hline OFDM Time Frame & \multicolumn{4}{|c}{$71 \times 10^{-2}$} \\
\hline Normalised Doppler frequency, $f_{d}^{\prime}$ & \multicolumn{4}{|c}{3072} \\
\hline $\begin{array}{l}\text { OFDM symbol normalised Doppler fre- } \\
\text { quency, } F_{D}\end{array}$ & 858 & 1536 & 3072 & 4272 \\
\hline FEC Coded Bits/Packet & $4.0 \mathrm{Mbps}$ & $7.2 \mathrm{Mbps}$ & $14.4 \mathrm{Mbps}$ & $20.0 \mathrm{Mbps}$ \\
\hline FEC-coded video bitrate & 427 & 766 & 1534 & 2134 \\
\hline No. of unprotected bits/packet & $2.0 \mathrm{Mbps}$ & $3.6 \mathrm{Mbps}$ & $7.2 \mathrm{Mbps}$ & $10.0 \mathrm{Mbps}$ \\
\hline Unprotected bitrate & 16 & 16 & 16 & 16 \\
\hline No. of CRC bits & 9 & 9 & 9 & 9 \\
\hline No. of feedback error flag bits & 10 & 11 & 12 & 13 \\
\hline No. of packet header bits/packet & 392 & 730 & 1497 & 2096 \\
\hline Effective video bits/packet & $1.8 \mathrm{Mbps}$ & $3.4 \mathrm{Mbps}$ & $7.0 \mathrm{Mbps}$ & $9.8 \mathrm{Mbps}$ \\
\hline Effective video bitrate & & BPSK & QPSK & \\
\hline Equivalent modulation mode & 8.8 & 11.0 & 16.1 & 19.2 \\
\hline Minimum channel SNR for 5\% PLR (dB) & 7.1 & 9.2 & 14.1 & 17.3 \\
\hline Minimum channel SNR for 10\% PLR (dB) & & & &
\end{tabular}

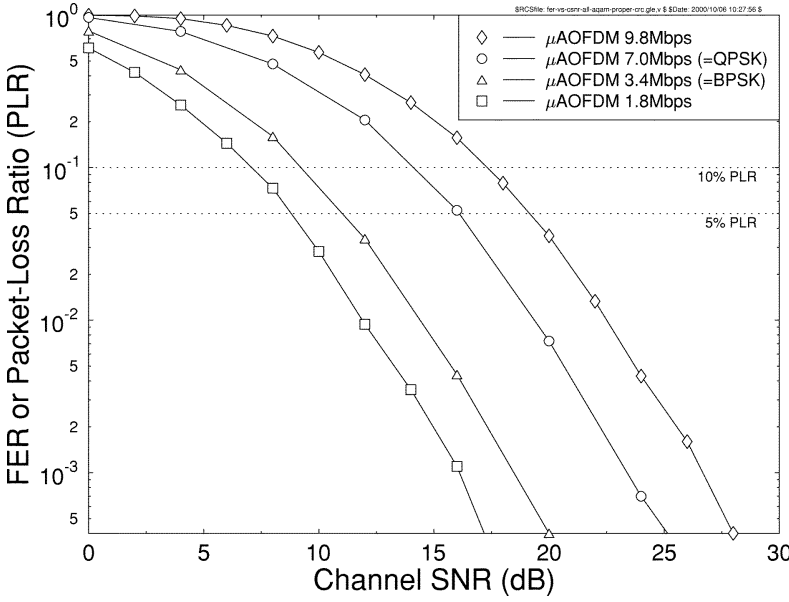

Fig. 6. FER or video PLR versus channel SNR for the subband-adaptive OFDM transceivers of Table II operating at four different target bit rates, over the channel model of Fig. 2 at a normalized Doppler frequency of $F_{D}=7.41 \times 10^{-2}$.

modulation mode transceivers of Table I. The system parameters for all four different bit rate modes are summarized in Table II. The effective video bit rates of 3.4 and 7.0 Mbits/s are equivalent to the bit rates of a fixed BPSK and QPSK mode transceiver, respectively.

Fig. 6 shows the transmission FER-not video FER-or video PLR performance versus channel SNR for the four different target bit rates of Table II. Fig. 6 demonstrates that-as expected - the higher target bit rate modes require higher channel SNRs in order to operate within the given PLR constraints. For example, the mode having an effective video bit rate of $9.8 \mathrm{Mbits} / \mathrm{s}$ can only operate for channel SNRs in excess of $19 \mathrm{~dB}$ under the constraint of a maximum PLR of $5 \%$. However, the mode having an effective video bit rate of 3.4 Mbits/s is capable of operating at channel SNRs of $11 \mathrm{~dB}$ and above while maintaining the same 5\% PLR constraint, albeit at about half the throughput bit rate, and hence at a lower video quality.

\section{TIME-VARIANT TARGET Bit RATE OFDM TRANSCEIVERS}

By using a high target bit rate when the channel quality is high, while aiming for a reduced target bit rate when the channel quality is poor, an adaptive system is capable of maximizing the average throughput bit rate over a wide range of channel SNRs, while maintaining a given quality constraint. This quality constraint for our video system could be the maximum PLR.

However, there is a substantial processing delay associated with evaluating the PLR, since averaging over a longer time interval would be necessitated. Hence, the employment of modem mode switching based on the PLR metric would be less efficient due to this latency. Therefore, we decided to invoke an estimate of the BER for mode switching, which can be generated as follows. Since the noise energy in each subcarrier is independent of the channel's frequency-domain transfer function $H_{n}$, the SNR estimate associated with the subcarrier $n$ can be expressed as

$$
\gamma_{n}=\left|H_{n}\right|^{2} \cdot \gamma
$$

where $\gamma$ is the overall SNR. ${ }^{4}$ If no signal degradation is imposed by inter-subcarrier interference (ISI) or interference due to other users, then the value of $\gamma_{n}$ determines the BEP for the transmission of data symbols over the subcarrier $n$. Given $\gamma_{j}$ across the $N_{s}$ subcarriers in the $j$ th subband, the expected overall BER for all available modulation schemes $M_{n}$ in each subband can be estimated, which is denoted by $\bar{p}_{e}(n)=1 / N_{s} \sum_{j} p_{e}\left(\gamma_{j}, M_{n}\right)$. For each subband, the specific modem mode having the highest throughput whose estimated BER was lower than the target BER was then activated for the subband concerned.

\footnotetext{
${ }^{4}$ The channel's transfer function $H_{n}$ can be estimated with the aid of sampling it using pilot subcarriers. The channel transfer function estimate can then be generated using sinc-function-aided interpolation between the pilot subcarriers, which generates a transfer function estimate for all subcarriers [1]. In these investigations, perfect channel transfer function estimation was used at the receiver and $H_{n}$ was assumed to be unchanged between the instant of estimation at the receiver and the allocation of the subband modes at the transmitter. As noted before, a better performance can be achieved, when employing more sophisticated channel transfer function predictors [32].
} 


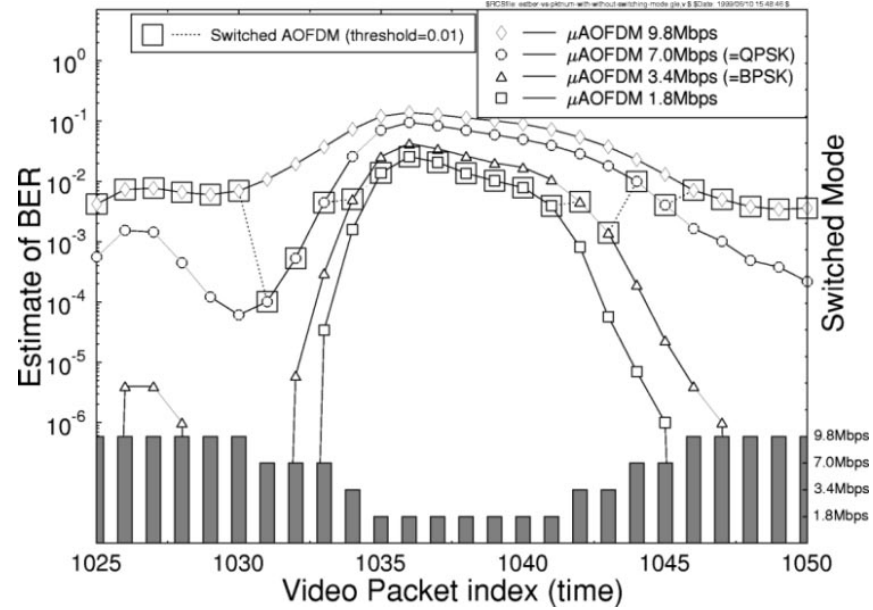

Fig. 7. Illustration of mode switching for the switched-subband adaptive modem. The figure shows the estimate of the BER for the four possible modes. The large square and the dotted line indicate the modem mode chosen for each time interval by the mode-switching algorithm. At the bottom of the graph, the bar chart specifies the bit rate of the switched subband adaptive modem on the right-hand axis versus time when using the channel model of Fig. 2 at a normalized Doppler frequency of $F_{D}=7.41 \times 10^{-2}$.

We decided to use a quadruple-mode-switched subband-adaptive modem using the four target bit rates of Table II. The channel estimator can then estimate the expected BER of the four possible modem modes. Again, the switching regime activated the specific modem mode whose estimated BER was below the required threshold. This threshold could be varied in order to tune the behavior of the switched subband-adaptive modem for a high or a low throughput. The advantage of a higher throughput was a higher error-free video quality at the expense of increased video packet losses, which may reduce the perceived video quality.

Fig. 7 demonstrates, how the switching algorithm operates for a $1 \%$ estimated BER threshold. Specifically, the figure portrays the estimate of the BER for the four possible modem modes versus time. The large square and the dotted line indicates the mode chosen for each time interval by the mode-switching algorithm. The algorithm attempts to use the highest bit-rate mode, whose BER estimate is less than the target threshold, namely $1 \%$ in this case. However, if all four modes' estimate of the BER is above the $1 \%$ threshold, then the lowest bit-rate mode is chosen, since this will be the most robust to channel errors. An example of this is shown around transmission frames or OFDFM symbol indices of 1035-1040. At the bottom of the graph, a bar chart specifies the bit rate of the switched-subband adaptive modem versus time, in order to emphasize when the switching occurs.

An example characterizing the algorithm, when switching among the target bit rates of $1.8,3.4,7$, and $9.8 \mathrm{Mbits} / \mathrm{s}$, is shown in Fig. 1. Fig. 1(a) portrays the contour plot of the channel SNR for each subcarrier versus time, while Fig. 1(d) displays the modulation mode chosen for each 32-subcarrier subband versus time for the time-variant target bit rate (TVTBR) subband adaptive modem. It can be seen at frames 1051-1055 that all the subbands employ QPSK modulation; therefore, the TVTBR-AOFDM modem has an instantaneous target bit rate of $7 \mathrm{Mbits} / \mathrm{s}$. As the channel degrades around frame 1060, the modem has switched to the more robust $1.8 \mathrm{Mbits} / \mathrm{s}$ mode.

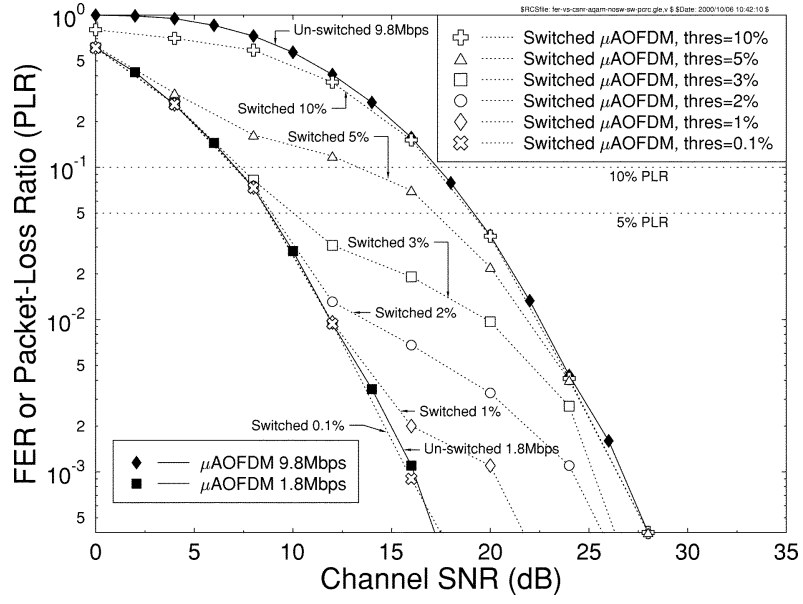

Fig. 8. FER or video PLR versus channel SNR for the TVTBR-AOFDM modem for a variety of BER switching thresholds. The switched modem uses four modes, with target bit rates of 1.8, 3.4, 7, and 9.8 Mbits/s. The unswitched 1.8 and $9.8 \mathrm{Mbits} / \mathrm{s}$ results are also shown in the graph as solid markers using the channel model of Fig. 2 at a normalized Doppler frequency of $F_{D}=7.41 \times 10^{-2}$.

When the channel quality is high around transmission frames 1074-1081, the highest bit rate $(9.8 \mathrm{Mbits} / \mathrm{s})$ mode is used. This demonstrates that the TVTBR-AOFDM modem can reduce the number of lost video packets by using reduced bit rate but more robust modulation modes, when the channel quality is poor. However, this is at the expense of a slightly reduced average throughput bit rate. Usually, a higher throughput bit rate results in a higher video quality; however, a high bit rate is also associated with a high PLR, which is usually less attractive in terms of perceived video quality than a lower bit rate, lower PLR mode.

Having highlighted how the time-domain mode-switching algorithm operates, we will now characterize its performance for a range of different BER switching thresholds. A low BER switching threshold implies that the switching algorithm is cautious about switching to the higher bit rate modes, and therefore the system performance is characterized by a low video PLR and a low throughput bit rate. A high BER switching threshold results in the switching algorithm attempting to use the highest bit rate modes in all but the worst channel conditions. This results in a higher video PLR. However, if the PLR is not excessively high, a higher video throughput is achieved.

Fig. 8 portrays the video PLR or transmission FER performance of the TVTBR-AOFDM modem for a variety of BER thresholds, compared to the minimum and maximum rate unswitched modes. It can be seen that for a conservative BER switching threshold of $0.1 \%$ the TVTBR-AOFDM modem has a similar PLR performance to that of the 1.8-Mbits/s nonswitched or constant target bit rate (CTBR) subband adaptive modem. However, as we will show, the throughput of the switched modem is always better or equal to that of the unswitched modem, and becomes far superior, as the channel quality improves. Observe in the figure that the "aggressive" switching threshold of $10 \%$ has a similar PLR performance to that of the 9.8-Mbits/s CTBR-AOFDM modem. We found that in order to maintain a PLR of below 5\%, the BER switching thresholds of $2 \%$ and $3 \%$ offered the best overall performance, 


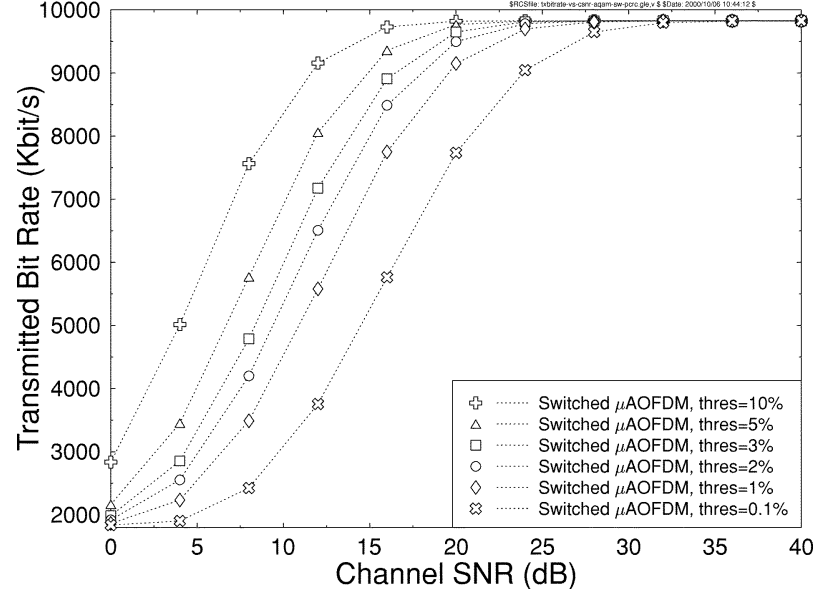

Fig. 9. Transmitted bit rate of the switched TVTBR-AOFDM modem for a variety of BER switching thresholds. The switched modem uses four modes, having target bit rates of $1.8,3.4,7$, and $9.8 \mathrm{Mbits} / \mathrm{s}$, over the channel model of Fig. 2 at a normalized Doppler frequency of $F_{D}=7.41 \times 10^{-2}$.

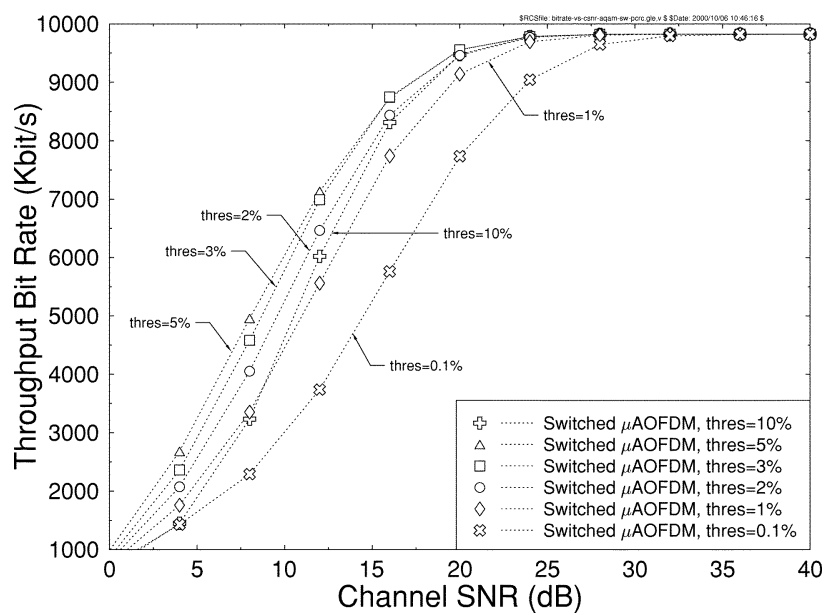

Fig. 10. Effective throughput bit rate of the switched TVTBR-AOFDM modem for a variety of BER switching thresholds. The switched modem uses four modes, with target bit rates of 1.8, 3.4, 7, and $9.8 \mathrm{Mbits} / \mathrm{s}$. The channel model of Fig. 2 is used at a normalized Doppler frequency of $F_{D}=7.41 \times 10^{-2}$.

since the PLR was fairly low, while the throughput bit rate was higher than that of an unswitched CTBR-AOFDM modem.

A high BER switching threshold results in the switched subband adaptive modem transmitting at a high average bit rate. However, we have shown in Fig. 8 how the PLR increases as the BER switching threshold is increased. Therefore the overall useful or effective throughput bit rate-i.e., the bit rate excluding lost packets-may in fact be reduced in conjunction with high BER switching thresholds. Fig. 9 demonstrates how the transmitted bit rate of the switched TVTBR-AOFDM modem increases with higher BER switching thresholds. However, when this is compared to the effective throughput bit rate, where the effects of packet loss are taken into account, the tradeoff between the BER switching threshold and the effective bit rate is less obvious. Fig. 10 portrays the corresponding effective throughput bit rate versus channel SNR for a range of BER switching thresholds. The figure demonstrates that for a BER switching threshold of $10 \%$ the effective throughput

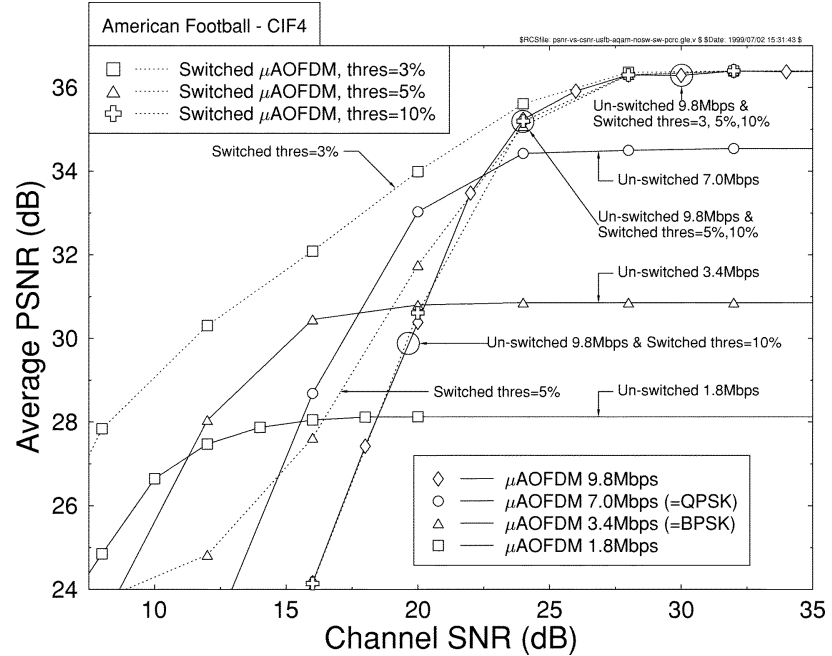

(a)

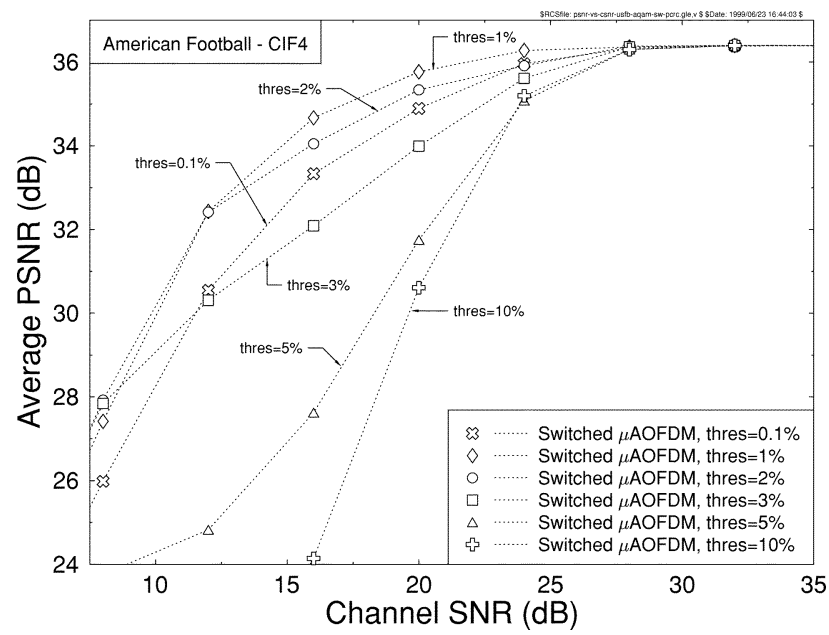

(b)

Fig. 11. Average PSNR versus channel SNR performance for switched- and unswitched subband adaptive modems. (a) Compares the four unswitched CTBR subband adaptive modems with switched TVTBR subband adaptive modems (using the same four modem modes) for switching thresholds of BER $=3 \%, 5 \%$, and 10\%. (b) Compares the switched TVTBR subband adaptive modems for switching thresholds of BER $=0.1 \%, 1 \%, 2 \%, 3 \%, 5 \%$, and $10 \%$.

bit-rate performance was reduced in comparison to some of the lower BER switching threshold scenarios. Therefore, the $\mathrm{BER}=10 \%$ switching threshold is obviously too aggressive, resulting in a high PLR and a reduced effective throughput bit rate. For the switching thresholds considered, the BER $=5 \%$ threshold achieved the highest effective throughput bit rate. However, even though the BER $=5 \%$ switching threshold produces the highest effective throughput bit rate, this is at the expense of a relatively high video PLR, which — as we will show-has a detrimental effect on the perceived video quality.

Fig. 11(a) compares the average PSNR versus channel SNR performance for a range of switched (TVTBR) and unswitched (CTBR) AOFDM modems. The figure compares the four unswitched, i.e., CTBR subband adaptive modems with switching, such as TVTBR subband adaptive modems, which switch between the four fixed-rate modes, depending on the BER switching threshold. The figure indicates that the switched TVTBR subband adaptive modem having a switching 
threshold of BER $=10 \%$ results in similar PSNR performance to the unswitched CTBR 9.8-Mbits/s subband adaptive modem. When the switching threshold is reduced to BER $=3 \%$, the switched TVTBR AOFDM modem outperforms all of the unswitched CTBR AOFDM modems. A switching threshold of $\mathrm{BER}=5 \%$ achieves a PSNR performance, which is better than the unswitched 9.8-Mbits/s CTBR AOFDM modem, but worse than that of the unswitched 7.0-Mbits/s modem, at low and medium channel SNRs.

A comparison of the switched TVTBR AOFDM modem employing all six switching thresholds that we have used previously is shown in Fig. 11(b). This figure suggests that switching thresholds of BER $=0.1 \%, 1 \%$, and $2 \%$ perform better than the $\mathrm{BER}=3 \%$ threshold, which outperformed all of the unswitched CTBR subband adaptive modems. The best average PSNR performance was achieved by a switching threshold of BER $=1 \%$. The more conservative BER $=0.1 \%$ switching threshold results in a lower PSNR performance, since its throughput bit rate was significantly reduced. Therefore, the best tradeoff in terms of PSNR, throughput bit rate, and video PLR was achieved with a switching threshold of about BER $=1 \%$.

\section{CONCLUSIONS}

A range of AOFDM video transceivers have been proposed for robust, flexible, and low-delay interactive video telephony. In order to minimize the amount of signaling required, we divided the OFDM subcarriers into subbands and controlled the modulation modes on a subband-by-subband basis. The proposed constant target bit rate AOFDM modems provided a lower BER than the corresponding conventional OFDM modems. The slightly more complex switched TVTBR-AOFDM modems can provide a balanced video quality performance, across a wider range of channel SNRs than the other schemes investigated. ${ }^{5}$

\section{ACKNOWLEDGMENT}

The constructive critique of the anonymous reviewers is gratefully acknowledged.

\section{REFERENCES}

[1] L. Hanzo, W. Webb, and T. Keller, Single- and Multi-Carrier Quadrature Amplitude Modulation. New York: IEEE Press/Wiley, Apr. 2000. [Online] Available: http://www-mobile.ecs.soton.ac.uk.

[2] T. Keller and L. Hanzo, "Adaptive multicarrier modulation: A convenient framework for time-frequency processing in wireless communications," Proc. IEEE, vol. 88, no. 5, pp. 611-642, May 2000.

[3] L. Hanzo, C. H. Wong, and M. S. Yee, Adaptive Wireless Transceivers: Turbo-Coded, Turbo-Equalised and Space-Time Coded TDMA, CDMA and OFDM Systems: Wiley/IEEE Press, 2002. [Online] Available: http://www3.interscience.wiley.com/cgi-bin.

[4] L. Hanzo, P. Cherriman, and J. Streit, Wireless Video Communications: From Second to Third Generation Systems, WLAN's and Beyond. New York: IEEE Press, 2001. [Online] Available: http://www-mobile.ecs.soton.ac.uk.

[5] R. Steele and W. Webb, "Variable rate QAM for data transmission over Rayleigh fading channels," Wireless, pp. 1-14, 1991.

[6] W. Webb and R. Steele, "Variable rate QAM for mobile radio," IEEE Trans. Commun., vol. 43, pp. 2223-2230, July 1995.

${ }^{5}$ Further information on research and publications can be found at http://www-mobile.ecs.soton.ac.uk/peter.
[7] H. Matsuoka, S. Sampei, N. Morinaga, and Y. Kamio, "Adaptive modulation system with variable coding rate concatenated code for high quality multi-media communications systems," in Proc. 46th Vehicular Technology Conf., 1996, pp. 478-491.

[8] S.-G. Chua and A. Goldsmith, "Variable-rate variable-power MQAM for fading channels," in Proc. 46th Vehicular Technology Conf., 1996, pp. 815-819.

[9] J. Torrance and L. Hanzo, "Latency and networking aspects of adaptive modems over slow indoors Rayleigh fading channels," IEEE Trans. Veh. Technol., vol. 48, pp. 1237-1251, 1998.

[10] J. Torrance, L. Hanzo, and T. Keller, "Interference aspects of adaptive modems over slow Rayleigh fading channels," IEEE Trans. Veh. Technol., vol. 48, pp. 1527-1545, Sept. 1999.

[11] V. K. N. Lau and M. D. Macleod, "Variable rate adaptive trellis coded QAM for high bandwidth efficiency applications in Rayleigh fading channels," in Proc. IEEE Vehicular Technology Conf., 1998, pp. 348-352.

[12] S. G. Chua and A. Goldsmith, "Adaptive coded modulation for fading channels," IEEE Trans. Commun., vol. 46, pp. 595-602, May 1998.

[13] T. Keller, J. Woodard, and L. Hanzo, "Turbo-coded parallel modem techniques for personal communications," in Proc. 47th Vehicular Technology Conf., Phoenix, AZ, May 5-7, 1997, pp. 2158-2162.

[14] K. Fazel, S. Kaiser, P. Robertson, and M. Ruf, "A concept of digital terrestrial television broadcasting," Wireless Pers. Commun., vol. 2, pp. 9-27, 1995.

[15] H. Sari, G. Karam, and I. Jeanclaude, "Transmission techniques for digital terrestrial tv broadcasting," IEEE Commun. Mag., pp. 101-1055, Feb. 1995.

[16] J. Borowski, S. Zeisberg, J. Hübner, K. Koora, E. Bogenfeld, and B. Kull, "Performance of OFDM and comparable single carrier system in MEDIAN demonstrator 60GHz channel," in Proc. ACTS Summit, Aalborg, Denmark, Oct. 1997, pp. 653-658.

[17] B. Engstroem and C. Oesterberg, "A system for test of multiaccess methods based on OFDM," in Proc. VTC'94, Stockholm, Sweden, 1994, pp. 1843-1847.

[18] E. Dahlman, B. Gudmundson, M. Nilsson, and J. Skold, "UMTS and IMT-2000 based on wideband CDMA," IEEE Commun. Mag., vol. 36, pp. 70-80, Sept. 1998

[19] Y. Li and N. R. Sollenberger, "Interference suppression in OFDM systems using adaptive antenna arrays," in Proc. Globecom 98, vol. 1, Sydney, Australia, Nov. 8-12, 1998, pp. 213-218.

[20] F. W. Vook and K. L. Baum, "Adaptive antennas for OFDM," in Proc. Vehicular Technology Conf., vol. 2. Ottawa, ON, Canada, May 18-21, 1998, pp. 606-610.

[21] J. S. Blogh and L. Hanzo, Third-Generation Systems and Intelligent Wireless Networking-Smart Antennas and Adaptive Modulation. New York: Wiley, 2002. [Online] Available: http://www3.interscience.wiley.com/cgi-bin, p. 408.

[22] L. Hanzo, F. Somerville, and J. Woodard, Voice compression and communications: Principles and applications for fixed and wireless channels, 2001. [Online] Available: http://www-mobile.ecs.soton.ac.uk.

[23] T. Willink and P. Wittke, "Optimization and performance evaluation of multicarrier transmission," IEEE Trans. Inform. Theory, vol. 43, pp. 426-440, Mar. 1997.

[24] L. Piazzo, "A fast algorithm for near-optimum power and bit allocation in OFDM systems," Electron. Lett., vol. 35, no. 25, pp. 2173-2174, Dec. 9, 1999.

[25] S. Lai, R. Cheng, K. Letaief, and R. Murch, "Adaptive trellis coded mqam and power optimization for ofdm transmission," in Proc. VTC'99. Houston, TX, May 16-20, 1999.

[26] D. Hughes-Hartogs, "Ensemble modem structure for imperfect transmission media," U.S. Patents 4679227 (July 1988) 4731816 (Mar. 1988) and 4833796 (May 1989).

[27] A. Czylwik, "Adaptive OFDM for wideband radio channels," in Proc. Globecom, London, U.K., 1996, pp. 713-718.

[28] R. F. H. Fischer and J. B. Huber, "A new loading algorithm for discrete multitone transmission," in Proc. Globecom, London, U.K., 1996, pp. 724-728.

[29] P. S. Chow, J. M. Cioffi, and J. A. C. Bingham, "A practical discrete multitone transceiver loading algorithm for data transmission over spectrally shaped channels," IEEE Trans. Commun., vol. 48, pp. 772-775, 1995 .

[30] S. Otsuki, S. Sampei, and N. Morinaga, "Square-qam adaptive modulation/TDMA/TDD systems using modulation estimation level with Walsh function," Electron. Lett., vol. 31, pp. 169-171, Feb. 1995.

[31] J. Torrance and L. Hanzo, "Demodulation level selection in adaptive modulation," Electron. Lett., vol. 32, pp. 1751-1752, Sept. 12, 1996 
[32] A. Duel-Hallen, S. Hu, and H. Hallen, "Long range prediction of fading signals," IEEE Signal Processing Mag., vol. 17, pp. 62-75, May 2000.

[33] I. Kalet, "The multitone channel," IEEE Tran. Commun., vol. 37, pp. 119-124, Feb. 1989.

[34] H. Rohling and R. Grünheid, "Performance of an OFDM-TDMA mobile communication system," in Proc. Globecom, London, U.K., 1996, pp. $1589-1593$.

[35] P. Cherriman and L. Hanzo, "Programmable H.263-based wireless video transceivers for interference-limited environments," IEEE Trans. Circuits Syst. Video Technol., vol. 8, pp. 275-286, June 1998.

[36] C. Berrou, A. Glavieux, and P. Thitimajshima, "Near Shannon limi error-correcting coding and decoding: Turbo codes," in Proc. Int. Conf. Communications, May 1993, pp. 1064-1070.

[37] L. Hanzo, T. Liew, and B. Yeap, Turbo Coding, Turbo Equalization and Space-Time Coding. New York: Wiley/EEE Press, 2002. [Online] Available: http://www-mobile.ecs.soton.ac.ukq.

Peter Cherriman received the M.Eng. degree in information engineering in 1994 from the University of Southampton, Southampton, U.K., where he is currently working toward the Ph.D. degree in mobile video networking

Since 1994, he was with the Department of Electronics and Computer Science, University of Southampton, working on projects for the Mobile Virtua Centre of Excellence. He joined the Research Department of the British Broadcasting Corporation (BBC), U.K., in 2001. His current areas of research include robust video coding, microcellular radio systems, power control, dynamic channel allocation, and multiple-access protocols.
Thomas Keller studied electrical engineering at the University of Karlsruhe, Ecole Superieure d'Ingenieurs en Electronique et Electrotechnique, Paris, France, and at the University of Southampton, Southampton, U.K. He received the Dipl.-Ing. degree in 1995 and the Ph.D. degree in 1999 with the Wireless Multimedia Communications Group, University of Southampton, in mobile communications.

Since 1999, he has been with Ubinetics, Cambridge, U.K., working on thirdgeneration CDMA system developments.

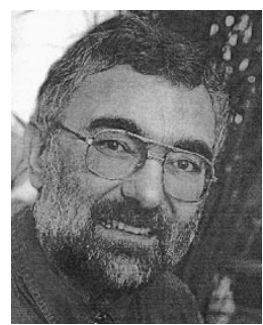

Lajos Hanzo received the M.Eng. degree in electronics in 1976 and the Ph.D. degree in 1983.

During his 24-year career in telecommunications, he has held various research and academic posts in Hungary, Germany, and the U.K. Since 1986, he has been with the Department of Electronics and Computer Science, University of Southampton, Southampton, U.K., where he currently holds the Chair in Telecommunications. He has coauthored eight books on mobile radio communications, published over 400 research papers, organized and chaired conference sessions, presented overview lectures, and has been awarded a number of distinctions. Currently, he is managing a research team, working on a range of research projects in the field of wireless multimedia communications under the auspices of the Engineering and Physical Science Research Council (EPSRC), U.K., the European IST Programme, and the Mobile Virtual Centre of Excellence (VCE). He is a Director of the VCE and also provides a range of industrial training courses.

Dr. Hanzo is an IEEE Distinguished Lecturer. 